Research Paper

\title{
Flooded Lung Generates a Suitable Acoustic Pathway for Transthoracic Application of High Intensity Focused Ultrasound in Liver
}

\author{
Thomas Günther Lesser ${ }^{1 凶}$, Carsten Boltze ${ }^{2}$, Harald Schubert ${ }^{3}$, and Frank Wolfram ${ }^{1}$ \\ 1. Department of Thoracic and Vascular Surgery, SRH Wald-Klinikum Gera, Teaching Hospital of Friedrich-Schiller University of Jena, Germany. \\ 2. Institute of Pathology, SRH Wald-Klinikum Gera, Teaching Hospital of Friedrich-Schiller University Jena, Germany. \\ 3. Institute of Animal Experimentation and Animal Welfare, Jena University Hospital, Friedrich-Schiller University Jena, Germany. \\ $\square$ Corresponding author: Thomas Günther Lesser, Department of Thoracic and Vascular Surgery, SRH Wald-Klinikum Gera, Teaching Hospital of \\ Friedrich-Schiller University of Jena, Strasse des Friedens 12207548 Gera, Germany. thomas.lesser@wkg.srh.de.
}

(1) Ivyspring International Publisher. Reproduction is permitted for personal, noncommercial use, provided that the article is in whole, unmodified, and properly cited. See http://ivyspring.com/terms for terms and conditions.

Received: 2016.06.07; Accepted: 2016.08.22; Published: 2016.09.19

\begin{abstract}
Background: In recent years, high intensity focused ultrasound (HIFU) has gained increasing clinical interest as a non-invasive method for local therapy of liver malignancies. HIFU treatment of tumours and metastases in the liver dome is limited due to the adjacent ultrasound blocking lung. One-lung flooding (OLF) enables complete sonography of lung and adjoining organs including liver. HIFU liver ablation passing through the flooded lung could enable a direct intercostal beam path and thus improve dose deposition in liver. In this study, we evaluate the feasibility of an ultrasound guided transthoracic, transpulmonary HIFU ablation of liver using OLF.

Methods: After right-side lung flooding, ultrasound guided HIFU was applied transthoracictranspulmonary into liver to create thermal lesions in three pigs. The HIFU beam was targeted five times into liver, two times at the liver surface and three times deeper into the tissue. During autopsy examinations of lung, diaphragm and liver located in the HIFU path were performed. The focal liver lesions and lung tissue out of the beam path were examined histologically.

Results: Fifteen thermal liver lesions were generated by transpulmonary HIFU sonication in all targeted regions. The lesions appeared well-demarcated in grey color with a cigar-shaped configuration. The mean length and width of the superficial and deeper lesions were $15.8 \mathrm{~mm}$ (range: $13-18 \mathrm{~mm}$ ) and $5.8 \mathrm{~mm}$ (range: $5-7 \mathrm{~mm}$ ), and $10.9 \mathrm{~mm}$ (range: $9-13 \mathrm{~mm}$ ) and $3.3 \mathrm{~mm}$ (range: 2-5 mm), respectively. Histopathological, all liver lesions revealed a homogeneous thermal necrosis lacking vitality. There were no signs of damage of the overlying diaphragm and lung tissue.

Conclusions: Flooded lung is a suitable pathway for applying HIFU to the liver, thus enabling a transthoracic, transpulmonary approach. The enlarged acoustic window could enhance the ablation speed for targets in the hepatic dome.
\end{abstract}

Key words: one-lung flooding, high intensity focused ultrasound, liver-ablation.

\section{Introduction}

About $75 \%$ of the patients with primary liver tumours and more than $80 \%$ of the patients with liver metastasis are not suited for surgical resection because of the associated extrahepatic disease, extent and location of the lesions in the liver, impaired liver function, or concurrent medical conditions [1,2]. Among the image-guided thermal ablation methods that are currently used to treat these nonsurgical patients, radiofrequency (RF) ablation is the most accepted technique. Therefore, a high rate of local tumour control $[3,4]$ comparable to stereotactic radiation can be achieved. Disadvantages are due to the substantial drop in the rate of complete ablation in tumours larger than $3 \mathrm{~cm}$ and in perivascular tumours 
by the heat sink effect [5]. Furthermore, the applicator's invasivity causes a potential risk for bleedings and tumour cell dissemination [6,7].

As an alternative, High Intensity Focused Ultrasound (HIFU) represents a completely non-invasive modality for local thermal tumour ablation without damaging the surrounding parenchymal tissue [8]. Several patient studies have investigated the feasibility of HIFU for the treatment of liver tumours or metastases [9,10,11], showing a strong therapeutic response to cancer tissue with almost negligible side effects. However, the tumour position affects its ability to be treated, for instance the location in the hepatic dome directly below the diaphragm [12,10]. The convex area of the liver is adjacent to the air filled lung representing an invincible border for diagnostic and therapeutic ultrasound [13]. As demonstrated in Figure 1, the shortest acoustic pathway transthoracically through the lung into the liver dome is currently impossible.

Furthermore, the gaseous lung needs to be protected from therapeutic ultrasound intensities causing tissue degeneration and ruptures at air tissue interfaces [14]. To overcome this problem and to enlarge the therapeutic window, pleural effusion is used for intercostal ultrasound [15] and MR guided HIFU [16]. This procedure seems to increase the therapeutic aperture and protecting lung tissue from HIFU.

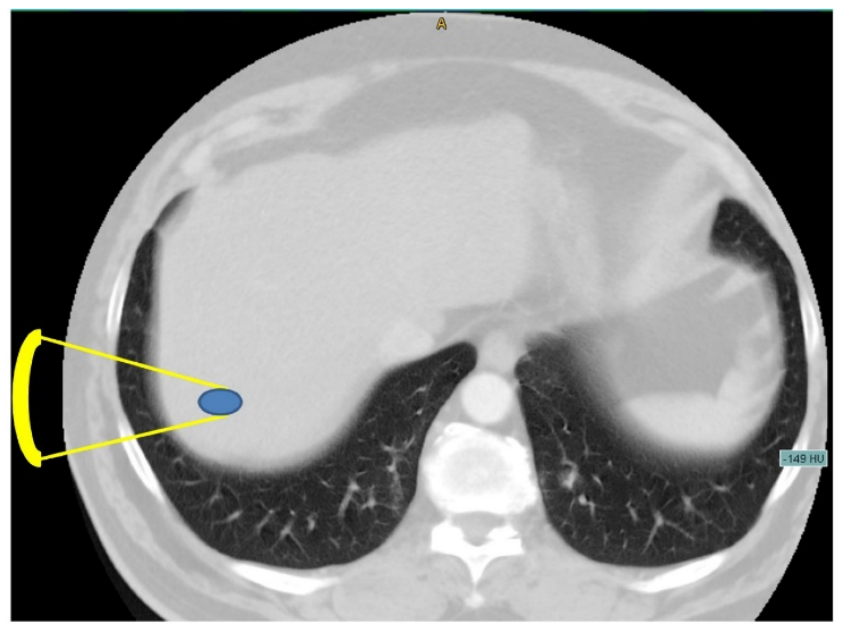

Figure 1. Axial CT image of the upper abdomen demonstrating the problem of a direct transpulmonary access to the liver. Shortest projection from a hypothetical extracostal transducer placement is indicated (yellow lines).

One-Lung Flooding (OLF) replaces air with saline content in one lung wing section enabling efficient lung sonography and reducing diaphragm motion in an in vivo porcine model $[17,18]$. Its safety has been shown on large animal models not causing disturbance of oxygenation, hemodynamic and surfactant outwash $[19,20]$. Beside the achieved sonographic access to lung and lung tumours, it was reported that adjacent organs can be imaged as well transpulmonary [21]. However, OLF was introduced to enable sonographic access to lung tumours for minimal-invasive surgery [22] and HIFU ablation of those [23]. It was found that flooded lung represents an ideal acoustic path with low attenuation for HIFU insonation into lung tumours [24]. Thus, it seems possible that right-sided OLF enables a direct acoustic path to the liver dome for HIFU ablation. In comparison to pleural effusion, OLF offers some advantages including lower invasivity, a position independent saline location for acoustic coupling and lower diaphragmatic movement [18].

The aim of this study was to assess the feasibility of a transthoracic, transpulmonary ablation of liver parenchyma using OLF in an in vivo large animal model.

\section{Materials and Methods}

\section{Animal preparation}

Animal experiments were performed in three female pigs (Deutsches Landschwein breed; weight: 27 to $33 \mathrm{~kg}$; average: $30.3 \mathrm{~kg}$ ). This study was carried out in strict accordance with the recommendations of the Guide for the Care and Use of Laboratory Animals in compliance with the National Animal Protection Act. The protocol was approved by the Committee on the Ethics of Animal Experiments of Veterinary Department of the Thuringian State Authority for Food Protection and Fair Trading (Thüringer Landesamt für Lebensmittelsicherheit und Verbraucherschutz, TLLV; Permit Number: 15-003/12). All surgery was performed under propofol and sufentanil anesthesia, and all efforts were made to minimize suffering.

The animals were premedicated with ketamine (1200 mg i.m.) and midazolam (10 mg i.m.), and general anaesthesia was induced with sufentanil $(1 \mu \mathrm{g}$ $\left.\mathrm{kg}^{-1}\right)$ and propofol $\left(3 \mathrm{mg} \mathrm{kg}^{-1}\right)$ via a peripheral vein. Anaesthesia was maintained with a continuous infusion of propofol $\left(10 \mathrm{mg} \mathrm{kg}^{-1} \mathrm{~h}^{-1}\right)$ and sufentanil $\left(0.02 \mu \mathrm{g} \mathrm{kg}^{-1} \mathrm{~min}^{-1}\right)$. After the onset of anaesthesia, the pigs were intubated with a single lumen endotracheal tube (Magill tube, inner diameter $=8.5 \mathrm{~mm}$, Mallinckrodt ${ }^{\mathrm{TM}}$, Covidien, Neustadt, Germany). The animals were relaxed (pancuronium bromide, $2.5 \mu \mathrm{g}$ $\mathrm{kg}^{-1} \mathrm{~min}^{-1}$ ) and mechanically ventilated with an intensive care unit (ICU) respirator (Servo 900, Siemens AG, Munich, Germany) on a volume-controlled setting $\left(\mathrm{FIO}_{2}=0.4\right.$; tidal volume 10 $\mathrm{ml} \mathrm{kg}{ }^{-1}$ body weight with the respiratory rate adjusted to maintain an end tidal $\mathrm{CO}_{2}$ of 35 to $45 \mathrm{mmHg}$; I:E ratio 1:1; PEEP $5 \mathrm{cmH}_{2} \mathrm{O}$ ). 
Following the tracheotomy, a left-sided Robertshaw double-lumen tube with an extra-long bronchial lane (size $39 \mathrm{Ch}$; special product by Mallinckrodt Medical, Dublin, Ireland) was inserted. The correct position of the tube was checked by fibrebronchoscopy (BF 3C30; Olympus, Tokyo, Japan). The right internal jugular vein was dissected, and a three- lumen central venous catheter (certofix; B. Braun, Melsungen, Germany) was inserted. The right carotid artery was cannulated (Arterial Leader Cath 2.7 Fr; Vygon, Ecouen, France) to allow blood gas analysis and invasive measurement of blood pressure. The electrocardiogram, arterial blood pressure, capillary oxygen saturation, and expiratory $\mathrm{CO}_{2}$ concentration were measured and recorded continuously (Datex AS/3 Patient Monitor; Datex-Ohmeda Corp., Helsinki, Finland). Arterial blood gases were analysed every 30 minutes (ABL System 625; Radiometer Medical, Copenhagen, Denmark).

Thirty minutes after ventilation with $\mathrm{FIO}_{2}=1.0$, the right endobronchial leg was disconnected from the respirator. The infusion system was immediately connected to the right tube leg and the lung was slowly filled with $465 \mathrm{ml}$ of degassed and tempered $\left(35^{\circ} \mathrm{C}\right)$ isotonic saline solution. This volume represents the functional residual capacity (FRC) and the tidal volume (TV) of a porcine lung wing [25]. A single filling was performed passively using the gravity of the liquid flowing from an infusion bottle suspended $50 \mathrm{~cm}$ above the heart level. During one-lung ventilation, the respirator settings remained unchanged.

Once the animals were placed in the lateral decubitus position (left lung below) two ribs overlying the right lower lung lobe were partially resected without opening the pleural cavity.

For re-ventilation the fluid of the right lung was partly drained passively out by Trendelenburg position. Double lung ventilation with the initial settings was performed for 30 minutes before scarification by injection of a lethal dose of sodium pentobarbital and potassium chloride.

\section{Sonography and HIFU application}

The flooded lung and the liver were sonographically examined using a diagnostic imaging system (bk 3000, BK Medical, Aarhus, DK). The self-manufactured HIFU applicator contained a HIFU transducer (SU102, Sonic Concepts Inc., Bothell, WA, USA) with a sideways attached sector probe (C11e, 5-8 MHz, SonoSite Inc, Bothell, WA, USA) (Figure $2 \mathrm{~A})$. The image plane was aligned with the central HIFU axis in order to position the focal region and to visualize the ablation zone. The surgical HIFU probe had an outer diameter of $35 \mathrm{~mm}$ with a focal depth of $55 \mathrm{~mm}$ and operated at 2,75 MHz. For each sonication a peak electric power of $75 \mathrm{~W}$ was applied to the HIFU transducer with a duty cycle of $50 \%$ (50 ms ON / 50 ms OFF), manually initiated for five seconds. The HIFU device was brought into contact with the costal pleura in the region of the resected ribs (Figure 2B) using an ultrasound coupling gel pad (Gelatin, BLOOM 260).

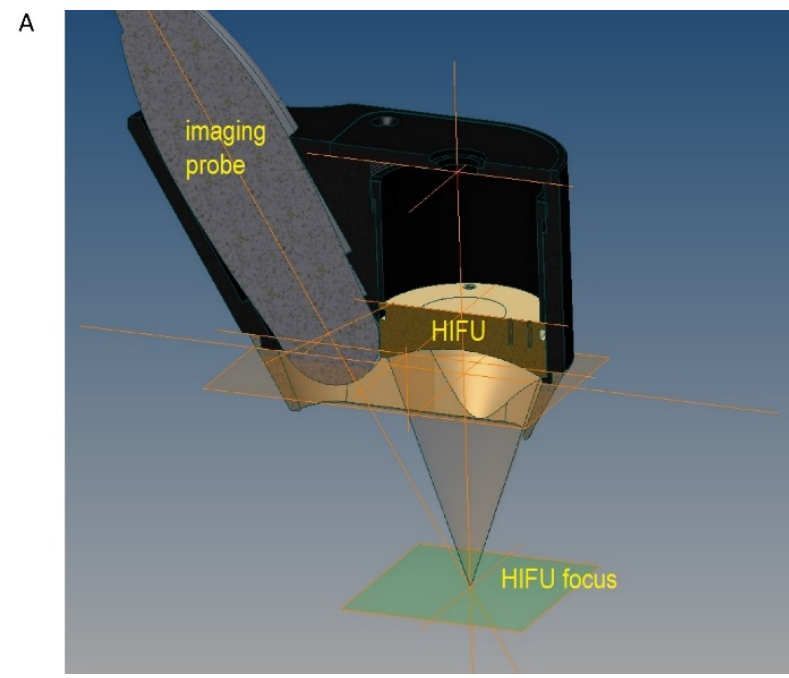

B

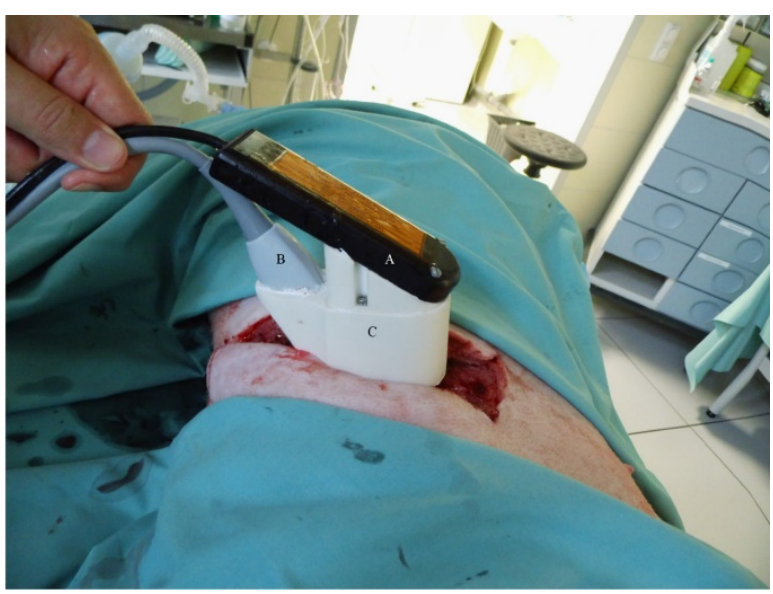

Figure 2. A) Schematic drawing of the HIFU applicator containing imaging probe and HIFU transducer with alignment of imaging plane and focal zone. B) in vivo transthoracic, transpulmonary application of high-intensity focused ultrasound to the liver after rib resection in a pig with a self-manufactured HIFU applicator.

Under imaging control, the HIFU focus was placed within the liver on a direct pathway through the flooded lung and the diaphragm. The liver tissue was exposed to HIFU for five seconds during inspirational breath hold for 15 seconds. The location of the focus was changed by slight tilting and pushing of the applicator. This procedure was repeated to create two thermal lesions at the liver surface and three lesions deeper within the liver. On one additional pig, HIFU was applied directly on liver using an AGAR-AGAR ( $2 \%$ by weight) coupling pad 
after surgical opening of the abdominal skin wall. Here five times the HIFU was targeted superficial and deep into liver. This experiment was performed to evaluate the lesion size based on a known low attenuation propagation medium (AGAR).

Immediately after HIFU exposure an ultrasound B-mode image was acquired. After the last HIFU insonation the beam path was marked by injection of methylene blue with a needle which was inserted in the liver along the acoustic axis under sonographic guidance. The dye was injected during retraction of the needle.

\section{Autopsy, histopathology and enzyme histochemistry}

Following euthanization, the thoracic and abdominal cavity was carefully opened to assess lung, diaphragm and liver macroscopically. The thermal liver lesions were evaluated by gross section in the region of colour marking. The greatest length and width of each lesion were measured. The thermal lesions were removed from surrounding normal liver tissue, halved in the middle of the longitudinal axis and sampled for histological and enzyme-histochemical examinations. Lung tissue outside and out of the marked HIFU beam path was also sampled. Histopathological examination was performed after ablated areas were fixed in $10 \%$ phosphate-buffered formaldehyde, embedded in paraffin, and stained with hematoxylin and eosin (HE). The pieces frozen in liquid nitrogen were used for the nicotinamide adenine dinucleotide phosphate $(\mathrm{NADPH})$-diaphorase staining.

\section{Results}

The liver could be examined sonographically trough the flooded lung in all animals. Thereby the lung appeared brighter in echogenity than the liver. In between liver and lung, the diaphragm was visible as an echogenic demarcation (Figure 3A). The flooded lung showed no signs of residual gas content in all animals. Immediately after HIFU insonation a hyperechoic region appeared in B-mode images at the focal position (Figure 3B). Their intensity decreased continuously but remained visible.

All 15 transpulmonary HIFU applications caused a thermal lesion corresponding to a success rate of $100 \%$. All five lesions were detectable in the liver at each animal (Figure 4). The mean length and width of the superficial lesions was $15.8 \mathrm{~mm}$ (range: 13 - $18 \mathrm{~mm}$ ) and $5.8 \mathrm{~mm}$ (range: 5 - $7 \mathrm{~mm}$ ), respectively. The mean length and width of the deep lesions was $10.9 \mathrm{~mm}$ (range: $9-13 \mathrm{~mm}$ ) and $3.3 \mathrm{~mm}$ (range: 2 - $5 \mathrm{~mm}$ ), respectively. HIFU lesions generated in direct acoustic contact to liver using
AGAR coupling pad revealed for superficial formations a length of $9.5 \mathrm{~mm}$ (range: $8-11 \mathrm{~mm}$ ) and width of $4.5 \mathrm{~mm}$ (range: $4-6 \mathrm{~mm}$ ), while deeper lesions had a length of $7 \mathrm{~mm}$ (range: $5.5-8 \mathrm{~mm}$ ) and width of $2.6 \mathrm{~mm}$ (range: $2-3.5 \mathrm{~mm}$ ). The lesion sizes are summarized in Table 1. Macroscopically, the lesions appeared brightly to the surrounding liver parenchyma as an elliptical, cigar-shaped form. There were no signs of mechanical or thermal damaging of the overlying diaphragm. In one case with the largest superficial HIFU lesion the diaphragm above the lesion showed low thermal damage but no signs of rupture or mechanical disintegration (Figure 5).

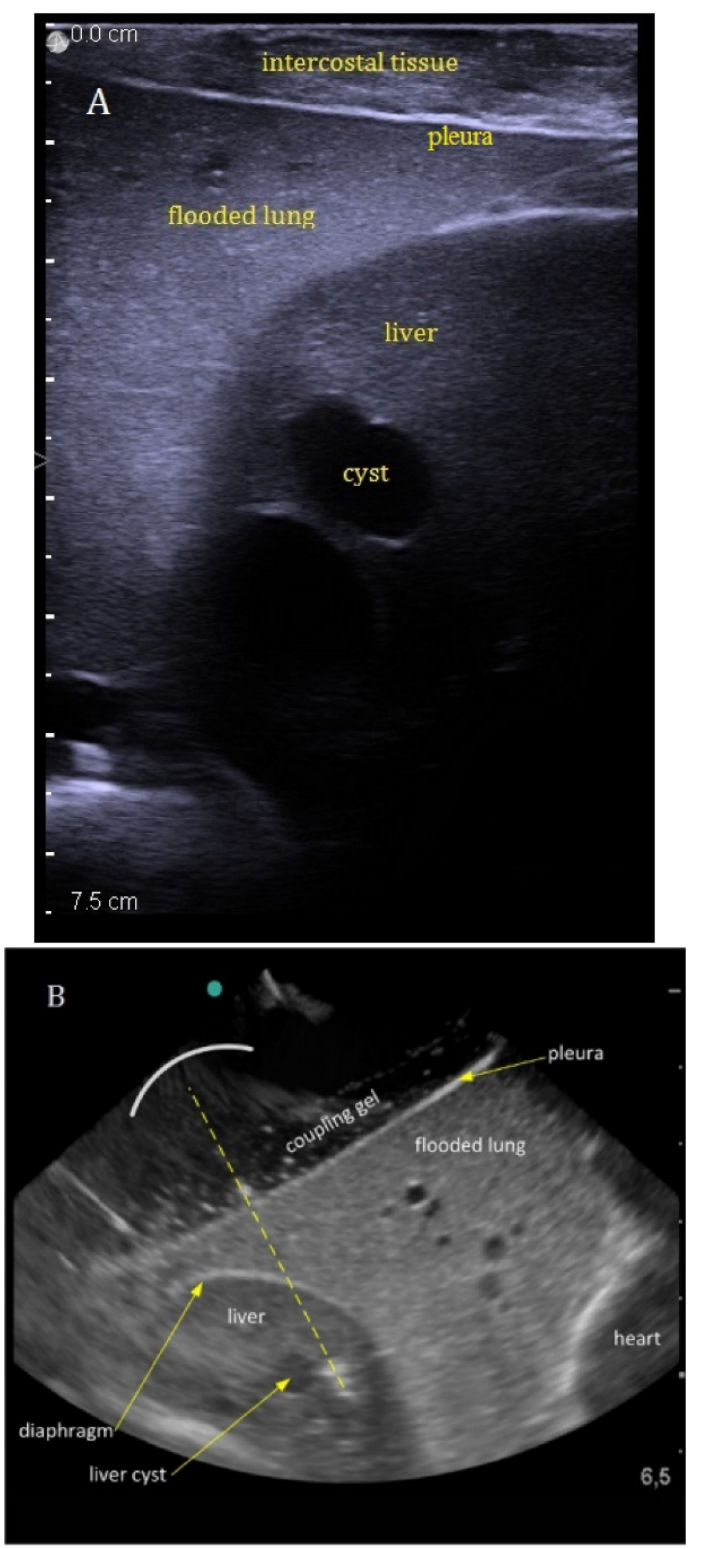

Figure 3. Sonographic imaging of the liver through the flooded lung before and after HIFU insonation. A) Transthoracic image shows subcutaneous tissue, pleura, flooded lung and liver. B) Immediately after HIFU insonation a well-demarcated hyperechoic sonolesion occurs in the liver. The yellow dashed line indicates the HIFU beam. 

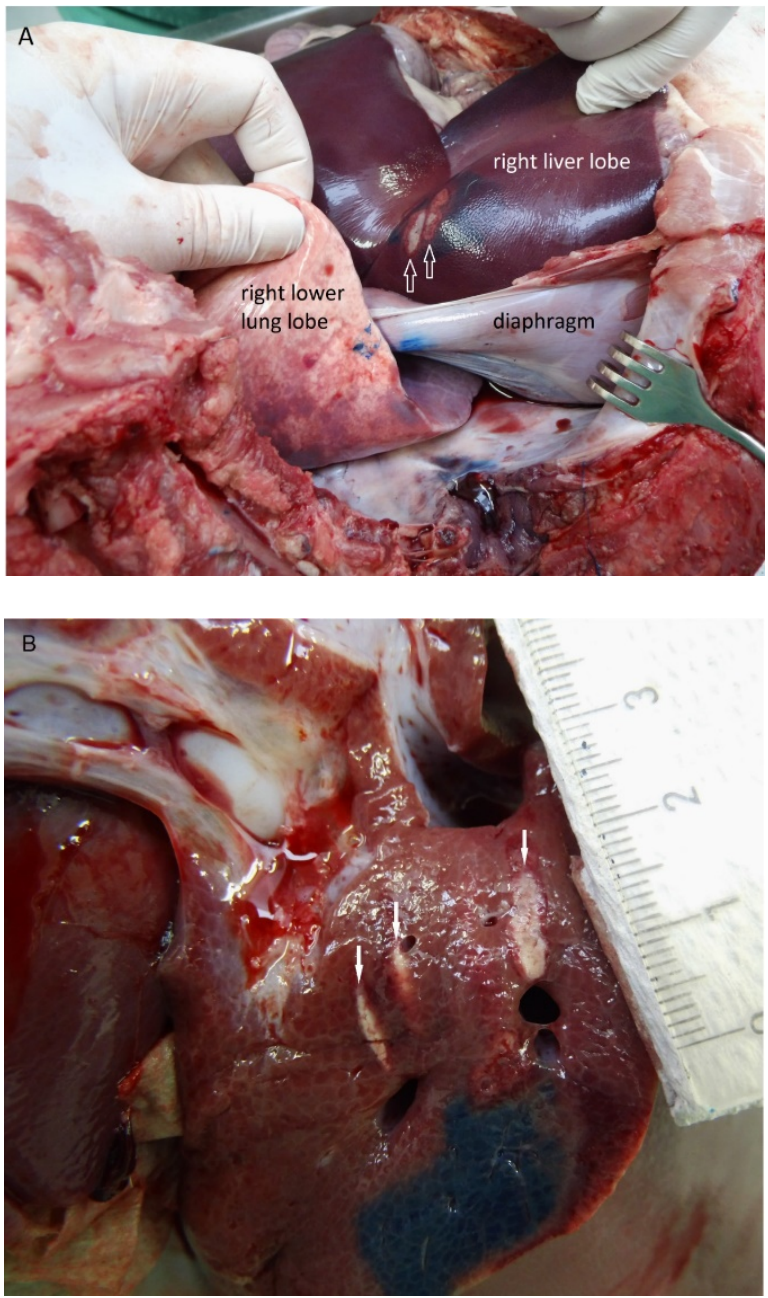

Figure 4. Macroscopic visualization of HIFU ablated areas in the liver. A) Typical well-demarked thermal lesions on the surface of the right liver lobe (arrows). The blue color marks the HIFU beam path through the thoracic wall, the lower lung lobe, the diaphragm. B) Three typical cigar-shaped thermal lesions deep in the liver parenchyma (arrows).

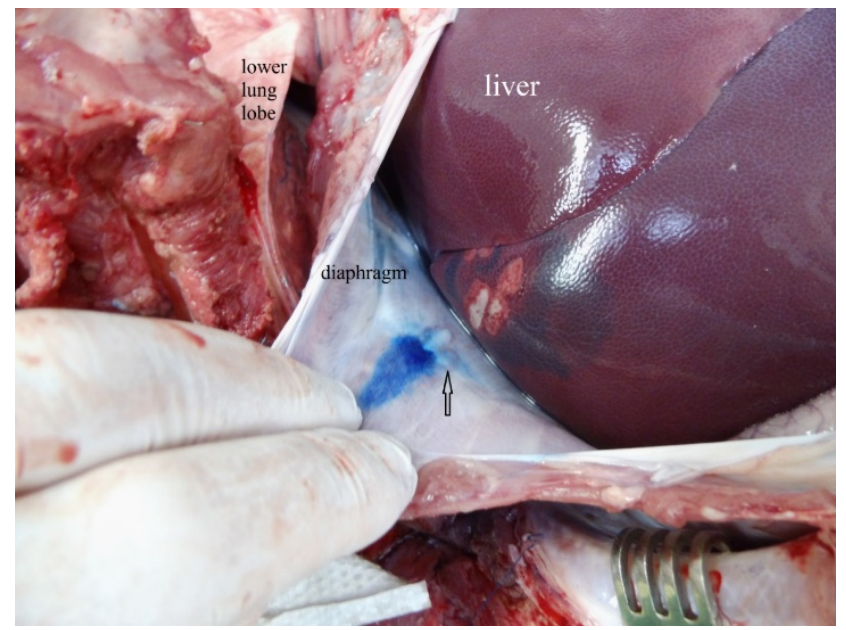

Figure 5. Macroscopic visualization of the abdominal diaphragm surface after transpulmonary, transdiaphragmatic HIFU insonation into the liver. Slightly thermal alteration of the parietal peritoneum of the diaphragm (arrow) opposite the liver lesions in the HIFU path (marked with blue color) in one case without signs of rupture or disintegration.
Table 1. Summary of lesion sizes induced by HIFU in liver, exposures were performed trans-pulmonary and in direct contact through AGAR coupling pad.

\begin{tabular}{llllllll}
\hline & & \multicolumn{3}{c}{ length $[\mathrm{mm}]$} & \multicolumn{3}{l}{ width [mm] } \\
\hline & mean & SD & range & mean & SD & range \\
trans-pulmonary & superficial & 15,83 & 1,72 & $13-18$ & 5,83 & 0,75 & $5-7$ \\
& deep & 10,89 & 1,54 & $9-13$ & 3,28 & 0,91 & $2-5$ \\
direct & superficial & 9,5 & 1,3 & $8-11$ & 4,5 & 0,6 & $4-6$ \\
AGAR & deep & 7 & 1,1 & $5,5-8$ & 2,6 & 0,5 & $2-3,5$ \\
\hline
\end{tabular}

The histological examination of all HIFU lesions confirmed coagulative necrosis of hepatocytes with cytoplasmic eosinophilia, disruption of cellular membranes with blurred cytoplasmic borders, karyolysis, and nuclear pyknosis in the center of the ablated area. There was a small region between the area of coagulation necrosis and vital liver tissue in which cells showed cytoplasmic vacuolization (Figure 6). A complete lack of NADPH diaphorase staining was seen in the ablated area, revealing homogeneous cell death with no areas of vitality (Figure 7A,B). Histopathological examination of the lung tissue out of the HIFU beam path showed no signs of damage as well as tissue not exposed to HIFU (Figure 8A,B).

All animals survived the procedure until the end point of $30 \mathrm{~min}$ two lung ventilation after saline draining without any signs of hypoxia or hypercapnia.

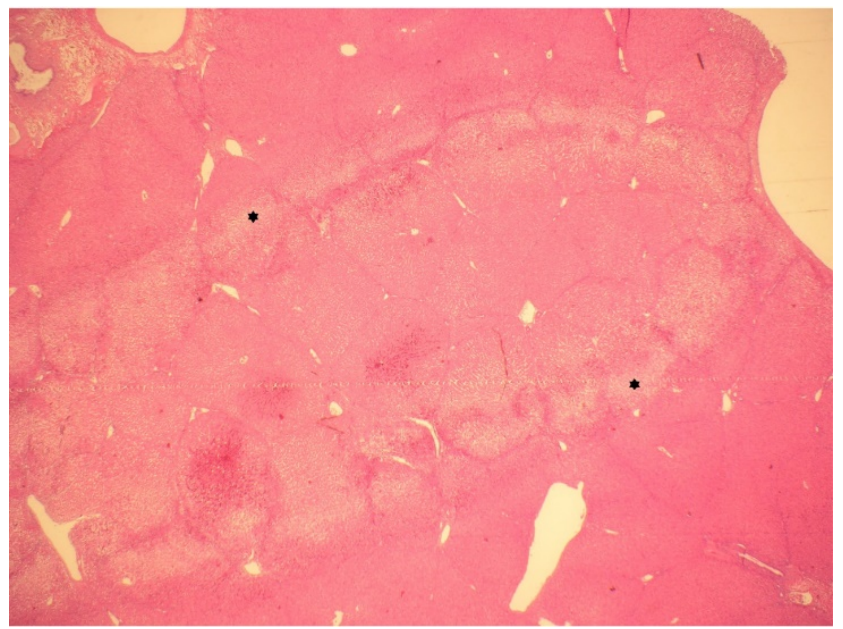

Figure 6. Histological examination of the liver after HIFU application. The thermal effect is visible as a coagulative necrosis with cytoplasmic eosinophilia, disruption of cellular membranes with blurred cytoplasmic borders, karyolysis, and nuclear pyknosis in the center as well as a small area, which contains cells with cytoplasmic vacuolization $(*)$ which represents a border between ablated and non-ablated liver tissue. H\&E, magnification $\times 10$ 


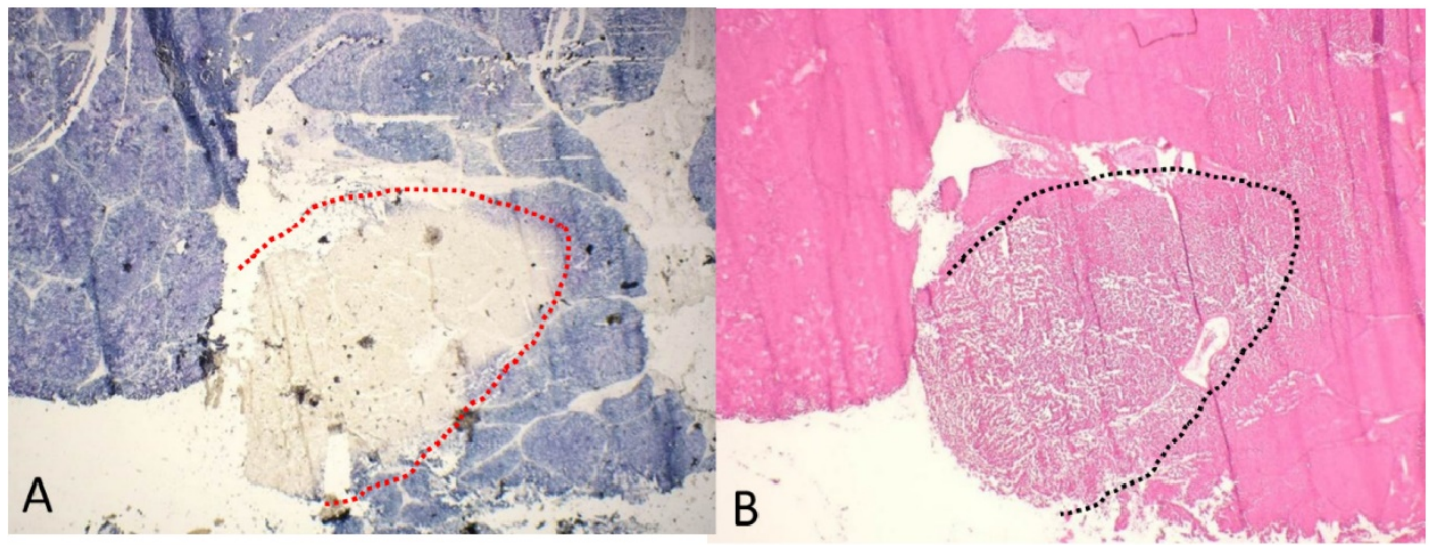

Figure 7. Enzyme histochemical examination after HIFU application at the liver surface. A) NADPH staining marks the non-ablated vital hepatocytes (blue color) with a sharp demarcation (dashed line) to the ablated area with the complete loss of vitality. B) Corresponding section stained with H\&E; both are frozen sections; magnification $\times 20$
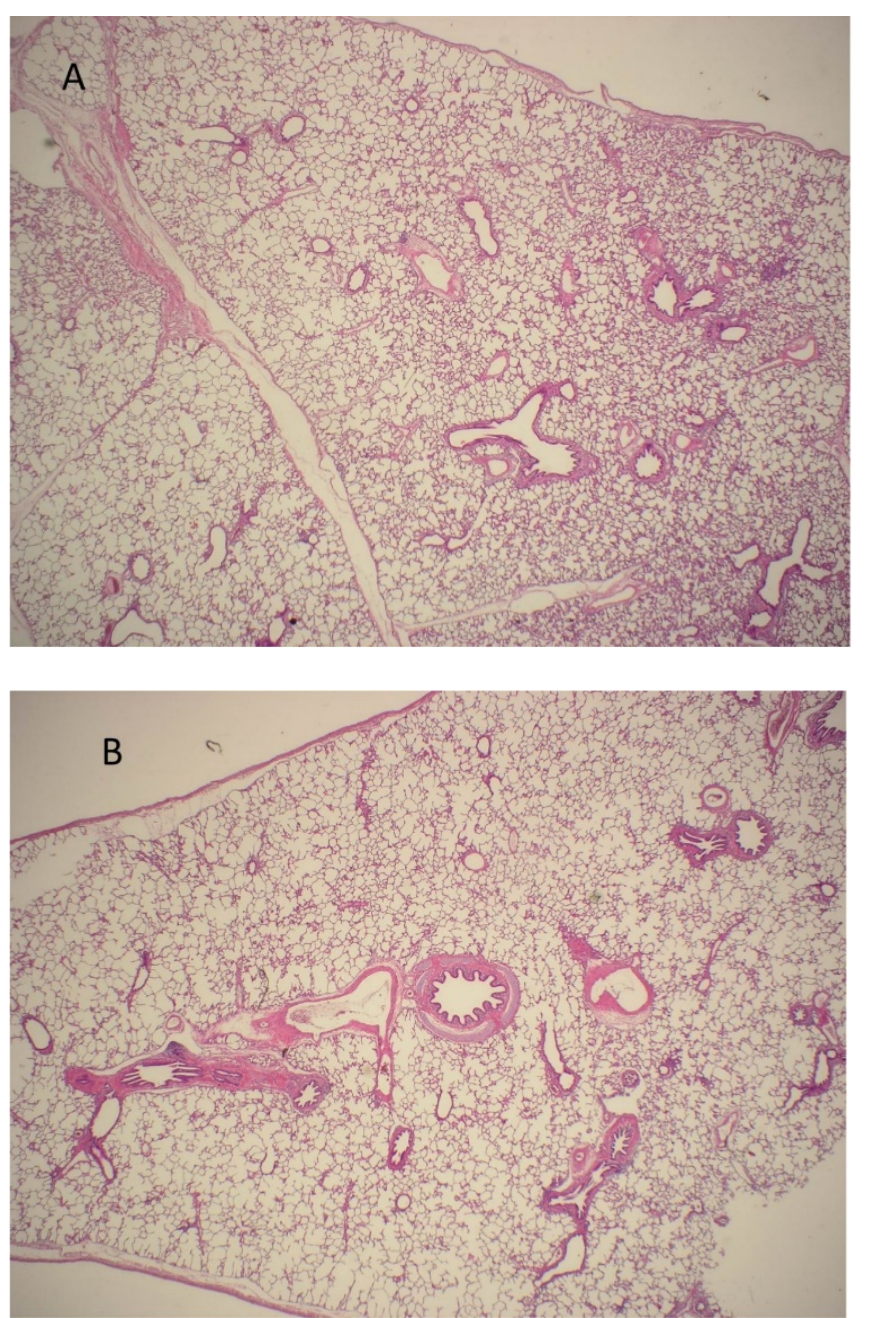

Figure 8. Histological examination of lung tissue outside (A) and out of the HIFU beam path (B) after HIFU application. There are no signs of structural damage of the lung tissue. H\&E, magnification $\times 10$.

\section{Discussion}

Our results demonstrate for the first time that transthoracic, transpulmonary HIFU ablation of liver tissue is feasible. All 15 HIFU applications caused relatively uniform thermal lesions, both in superficial and deep regions of the liver. After each HIFU exposure a bright hyperechoic region at the focal location occurred. This typical sign of ultrasound guided HIFU indicates a temperature rise above $80^{\circ} \mathrm{C}$ and vaporization in tissue [26]. The macroscopic and histopathological analysis showed coagulative necrosis of the same elliptical shape. The length and width of the deep lesions were smaller than the superficial lesions, which is plausible since tissue attenuate at $3 \mathrm{MHz}$ (HIFU frequency) significantly intensities. By passing $2 \mathrm{~cm}$ roughly $30 \%$ of the original HIFU intensity is absorbed.

The design of the intercostal applicator required a truncation of the spherical transducer, which causes a widening of the focal beam profile. The resulting acoustic window of $35 \mathrm{~mm} \times 12 \mathrm{~mm}$ was designed to fit to the width of a resected rib segment. Therefore, it cannot be referred to the focal beam profile based on manufacturers transducer specifications.

It is significant that lesions generated in direct applicator contact (AGAR) are smaller in length and width than transpulmonary. During transpulmonary HIFU the liver undergoes slight movements caused by cardiac action. This will be suppressed through the mechanical fixation when the AGAR pad is applied in contact to liver. The enlarged lesions size during transpulmonary HIFU in comparison to direct AGAR contact are caused by the truncation of HIFU aperture and blurring of HIFU intensity by cardiac motion.

HIFU lesions generated in liver with the same applicator on a direct acoustic access using an AGAR coupling pad showed similar appearance and shape as applied transpulmonary. This indicates that the focal energy deposition undergoes transpulmonary very low losses since AGAR is a low attenuating ultrasound coupling medium [27].

Flooded lung represents an ideal acoustic medium. The high quality of sonographic images of 
liver indicates an interference free acoustic transpulmonary access. Own measurements of acoustic properties have shown that flooded lung parenchyma has a very low attenuation (0.12 $\mathrm{dB} / \mathrm{cm} / \mathrm{MHz}$ ) [24]. The high water content serves as an acoustic conductor and dominates the characteristics of flooded lung. Without essential loss ultrasound waves passes through the flooded lung. Sound reflections at tissue interfaces (flooded lung/ diaphragm/ liver) are insignificant, because the acoustic impedance of these tissues is similar (1.6/1.65-1.74/1.66 MRayl) [28]. The low attenuation of flooded lung without impedance mismatch between the HIFU device and the liver are ideal conditions for an optimal energy transport and therefore heat induction in the liver.

In comparison to pleural effusion with saline, the liquid in flooded lung is statically trapped in the porous lung parenchyma. Therefore, acoustic streaming will be suppressed. It is likely that cavitation bubbles in the instilled intra-pleural liquid are induced and pushed by radiation forces towards the diaphragm. This could cause pre-focal shielding and is probably responsible for the reported diaphragm rupture during liver HIFU treatment [15]. Furthermore, the flooded lung, as a dense structure, stabilizes the liver in its anatomic position whereas with pleural effusion the liquid fills the pleural cavity depending on gravitation in an unhindered way. OLF might also offer systemic advantages because flooded lung is not perfused [19] and therefore suppresses pulmonary shunting. Depending on the ventilation conditions during pleural effusion, perfusion of the lung on the effused side may cause a shunt situation [29]. This might worsen the systemic gas exchange and effect patients with poor cardio-pulmonary function.

Histopathological examinations showed no damage of lung tissue in the pre-focal beam path. This was about to be expected, since HIFU exposure in flooded lung parenchyma results in much lower heat induction $(5-10 \mathrm{~K})$ than in solid lung cancer tissue (30 - 40 K) [24]. However, it seems possible that high ultrasound intensity generates cavitation, particularly in the saline component. Further investigations are planned to determine the effects of cavitation in flooded lung parenchyma.

The current ablation speed of MR guided FUS systems is insufficient to serve major requirements in hepatic oncology [30]. The shielding of the rib cage, target motion and the high perfusion in liver makes the use of fast volumetric ablation schemes not applicable. OLF provides a superior direct acoustic pathway to liver, enlarging the therapeutic window and reducing the liver motion. It needs to be discussed whether the advantages for a transpulmonal liver ablation justifies the increased procedural efforts of OLF.

Our study suffers from some limitations. A relative low number of animals have been used. Since 15 times HIFU successful induced necrotic lesions the results become valid. The rib cage represents a significant obstacle in transthoracic HIFU application [31,32]. The problem has been scientifically explored and methods for focusing through the rib cage have been demonstrated by using phased array transducers [33,34,35]. The self-manufactured HIFU device used in this study contained a single element transducer with an acoustic window of $35 \mathrm{~mm} \times 12 \mathrm{~mm}$. It required partial resection of two ribs to arrange complete acoustic access of the applicator with the pleura. In future clinical practice this resection would not be necessary by using clinical HIFU systems that can focus through the rib cage.

For proper location of the HIFU applicator, a lateral position was chosen where the flooded lung is above the ventilated one. During mechanical ventilation of the contralateral lung, the liver undergoes a rotation below the diaphragm which explains why a short breath-hold was necessary during HIFU insonation. In clinical practice, especially for MR guided HIFU, the flooded lung would be located reversed on the downside. Here the diaphragmatic motion is being significantly reduced during mechanical ventilation [18] with no need for forced respiratory maneuvers.

The aim of the study was to demonstrate that HIFU passes through flooded lung. We demonstrated that a single HIFU sonication of 5 seconds generates transpulmonary a thermal lesion in the liver dome. Further studies are needed to prove the efficacy of transthoracic, transpulmonary HIFU during ablation of liver tumours.

\section{Conclusion}

OLF generates a suitable acoustic pathway for transthoracic, transpulmonary HIFU application in liver. Thus, it seems possible to use the enlarged acoustic window provided by flooded lung whereby the efficacy of HIFU ablation in liver may be improved.

\section{Abbreviations}

HIFU: high-intensity focused ultrasound; OLF: one-lung flooding; FUS: Focused Ultrasound Surgery; PEEP: positive end expiratory pressure; $\mathrm{FIO}_{2}$ : fraction of inspired oxygen; ECG: electrocardiography; TV: tidal volume. 


\section{Acknowledgements}

The authors would like to thank Dr. Bischoff3, Mrs. Petra Dobermann ${ }^{3}$ as well as Dr. Ziemann and Dr. Pölzing (FIM, Beichlingen Germany) for help with the animal experiments and Mr. Rosenberger for hands on HIFU applicator design. This research was supported by the SRH Waldklinikum Gera, Germany; and the FUS Foundation, Charlottesville, VA, USA.

\section{Authors' contributions}

TGL collected and evaluated the data and wrote the manuscript. HS performed the anaesthesia and ventilation. FW was responsible for ultrasound imaging and HIFU technique. He co-wrote and revised the manuscript and discussed the results with the authors. CB performed the histopathological and enzyme histochemistry examinations. All authors read and approved the manuscript.

\section{Competing interests}

The authors declare that they have no competing interests, neither financial nor non-financial.

\section{References}

1. Bruix J, Sherman M. Management of hepatocellular carcinoma: an update. Hepatology. 2011; 53: 1020-2

2. Adams R, Aloia T, Loyer E, Pawlik T, Taouli B, Vauthey J. Selection for hepatic resection of colorectal liver metastases: expert consensus statement. HPB Oxf. 2013; 15: 91-103.

3. Gervais D, Goldberg S, Brown D, Soulen M, Millward S, Rajan D. Society of Interventional Radiology position statement on percutaneous radiofrequency ablation for the treatment of liver tumours. J Vasc Interv Radiol. 2009; 20: 342-7.

4. Salmi A, Turrini R, Lanzani G, Savio A, Anglani L. Efficacy of radiofrequency ablation of hepatocellular carcinoma associated with chronic liver disease without cirrhosis. Int J Med Sci. 2008; 5: 327-32.

5. Lencioni R, Crocetti L. Loco-regional treatment of hepatocellular carcinoma. Radiology. 2012; 262: 43-58.

6. Snoeren N, Huiskens J, Rijken A, van Hillegersberg R, van Erkel A, Slooter G, et al. Viable tumour tissue adherent to needle applicators after local ablation: a risk factor for local tumour progression. Ann Surg Oncol. 2011; 18: 3702-10.

7. Schuld J, Richter S, Oberkircher L, Seeland U, Debnar-Daumler K, Rauch J, et al. Evidence for tumour cell spread during local hepatic ablation of colorectal liver metastase. J Surg Res. 2012; 178: 268-79.

8. Kennedy JE, ter Haar G, Cranston D. High intensity focused ultrasound: surgery of the future? The British Journal of Radiology. 2003; 76: 590-9.

9. Illing R, Kennedy J, Wu F, ter Haar G, Protheroe A, Friend P, et al. The safety and feasibility of extracorporeal high-intensity focused ultrasound (HIFU) for the treatment of liver and kidney tumours in a Western population. Br J Cancer. 2005; 93: 890-5.

10. Wu F, Wang Z, Chen W, Zou J, Bai J, Zhu H, et al. Extracorporeal High-Intensity Focused Ultrasound for treatment of solid carcinomas. Ultrasound Med Biol. 2002; 30: 245-60.

11. Dupré A, Melodelima D, Pérol D, Chen Y, Vincenot J, Chapelon J, et al. First clinical experience of intra-operative high intensity focused ultrasound in patients with colorectal liver metastases: a phase I-IIa study. PLoS One. 2015; 10: e0118212. doi: 10.1371/journal.pone.0118212.eCollection 2015.

12. Al-Bataineh $\mathrm{O}$, Jenne J, Huber P. Clinical and future applications of high intensity focused ultrasound in cancer. Cancer Treat Rev. 2012; 38: 346-55.

13. Dunn F, Fry F. Ultrasonic Absorption and Reflexion by Lung Tissue. Phys Med Biol. 1961; 148: 401-10.

14. Simon J, Sapozhnikov O, Khokhlova V, Wang Y, Crum L, Bailey M. Ultrasonic atomization of tissue and its role in tissue fractionation by high intensity focused ultrasound. Phys Med Biol. 2012; 57: 8061-78.

15. Jung S, Cho S, Jang J, Han J. High-intensity focused ultrasound ablation in hepatic and pancreatic cancer: complications. Abdom Imaging. 2011; 36: $185-95$

16. Wijlemans J, de Greef M, Schubert G, Moonen C, van den Bosch M, Ries M. Intrapleural fluid infusion for MR-guided high-intensity focused ultrasound ablation in the liver dome. Acad Radiol. 2014; 21: 1597-1612.
17. Lesser $\mathrm{T}$, Klinzing $\mathrm{S}$, Schubert $\mathrm{H}$, Klein $\mathrm{U}$, Bartel M. Lung flooding--a new method for complete lung sonography. Resp Exp Med. 1998; 198: 83-91.

18. Lesser T, Schubert H, Güllmar D, Reichenbach J, Wolfram F. One-lung flooding reduces the ipsilateral diaphragm motion during mechanical ventilation. Eur J Med Res. 2016; 21: 9-14.

19. Klinzing S, Lesser T, Schubert H, Bloos F, Klein U, Bartel M. Hemodynamics and gas exchange during experimental one-lung fluid flooding in pigs. Res Exp Med. 1999; 199: 87-94.

20. Klinzing S, Lesser T, Schubert H, Bartel M, Klein U. Wet to Dry ratio of lung tissue and surfactant outwash after one lung flooding. Eur J Med Res. 2000; 200: 27-33.

21. Lesser $T$, Schubert $H$, Bischoff $S$, Wolfram F. Lung flooding enables efficient lung sonography and tumour imaging in human ex vivo and porcine in vivo lung cancer models. Eur J Med Res. 2013; 18: 23.

22. Klinzing S, Lesser T, Schubert H, Bartel M, Klein U. One lung flooding for video-assisted thoracoscopic surgery in animal experiments on pigs. Resp Exp Med. 2000; 199: 333-7.

23. Wolfram F, Boltze C, Schubert H, Bischoff S, Lesser T. Effect of lung flooding and high-intensity focused ultrasound on lung tumours: an experimental study in an ex vivo human cancer model and simulated in vivo tumours in pigs. Eur J Med Res. 2014; 19: 1.doi: 10.1186/2047-783X-19-1.

24. Wolfram F, Reichenbach J, Lesser T. An ex vivo human lung model for ultrasound guided HIFU therapy using lung flooding. Ultrasound in Med \& Biol. 2013; 40: 496-503.

25. Defosse J. Einfluss von Spontanatmung auf die endexspiratorische Belüftung der Lunge bei tierexperimentellem Lungenschaden. Dissertation, Bonn, Germany; 2012

26. Yang R, Nerendra T, Sanghvi T, Rescorla F, Galliani C, Fry F, et al. Extracorporeal Liver Ablation using sonography-guided High Intensity Focused Ultrasound. Investigative Radiology. 1992; 27: 796-803.

27. Sun C, Pye SD, Browne JE, Janeczko A, Ellis B, Butler MB, Sboros V, Thomson $\mathrm{AJ}$, Brewin MP, Earnshaw $\mathrm{CH}$, Moran CM. The speed of sound and attenuation of an IEC agar-based tissue-mimicking material for high frequency ultrasound applications. Ultrasound Med Biol. 2012, 38: 1262-70

28. Goss S, Johnston R, Dunn F. Comprehensive compilation of empirical ultrasonic properties of mammalian tissues. J Acoust Soc Am. 1978; 64: 423-57.

29. Cheung TT, Chu FS, Jenkins CR, Tsang DS, Chok K, Chan AC, et al. Tolerance of High-Intensity Focused Ultrasound Ablation in Patients with Hepatocellular Carcinoma. World J Surg. 2012; 36: 2420-7.

30. Wijlemans J, de Greef M, Schubert G, Bartels L, Moonen C, van den Bosch M, et al. A clinically feasible treatment protocol for magnetic resonance-guided high-intensity focused ultrasound ablation in the liver. Invest Radiol. 2015; 50: 24-31.

31. Lin J, Liu X, Zhang D, Gong X. Influence on ribs of the nonlinear sound field of therapheutic ultrasound. Ultrasound Med Biol. 2007; 33: 1413-20.

32. Salomir R, Petrusca L, Auboiroux V, Muller A, Vargas M, Morel D, et al. Magnetic resonance-guided shielding of prefocal acoustic obstacles in focused ultrasound therapy: application to intercostal ablation in liver. Invest Radiol. 2013; 48: 366-80

33. Aubry F, Pernot M, Marquet F, Tanter M, Fink M. Transcostal high-intensity-focused ultrasound: ex vivo adaptive focusing feasibility study. Phys Med Biol. 2009; 53: 2937-51.

34. Bobkova A, Gavrilov L, Khokhlova V, Shaw A, Hand J. Focusing of high-intensity ultrasound through the rib cage using a therapeutic random phased array. Ultrasound Med Biol. 2010; 36: 888-906.

35. Civale J, Clarke R, Rivens I, ter Haar G. The use of a segmented transducer for rib sparing in HIFU treatments. Ultrasound Med Biol. 2006; 32: 1753-61. 